\title{
Erratum
}

\section{Stability of a cloned gene in yeast grown in chemostat culture}

Richard M. Walmsley, David C.J. Gardner, and Stephen G. Oliver

Department of Biochemistry and Applied Molecular Biology, University of Manchester, Institute of Science and Technologie, P.O. Box 88, Manchester M60 1QD, UK

Mol Gen Genet (1983) 192:361-365

On page 362 at the end of the paragraph starting "F1 synthetic minimal medium ...", the constituent " $20 \mathrm{~g}$ glucose" should read " 2 g glucose". 